\title{
ELEMENTARY MECHANICS OF THE MITRAL VALVE*
}

\author{
D. AMBROSI ${ }^{\dagger}$, L. DEORSOLA ${ }^{\ddagger}$, S. TURZI§, AND M. ZOPPELLO ${ }^{\dagger}$
}

\begin{abstract}
We illustrate a bare-bones mathematical model that is able to account for the elementary mechanics of the mitral valve when the leaflets of the valve close under the systolic pressure. The mechanical model exploits the aspect ratio of the valve leaflets that are represented as inextensible rods, subject to the blood pressure, with one fixed endpoint (on the endocardium) and an attached wire anchored to the papillary muscle. Force and torque balance equations are obtained exploiting the principle of virtual work, where the first contact point between rods is identified by the Weierstrass-Erdmann condition of variational nature. The chordae tendineae are modeled as a force applied to the free endpoint of the flaps. Different possible boundary conditions are investigated at the mitral annulus, and, by an asymptotic analysis, we demonstrate that in the pressure regime of interest generic boundary conditions generate a tensional boundary layer. Conversely, a specific choice of the boundary condition inhibits the generation of high tensional gradients in a small layer.
\end{abstract}

Key words. mitral valve, mechanics, rod, contact, asymptotic analysis

AMS subject classification. $74 \mathrm{~L} 15$

DOI. $10.1137 / 21 \mathrm{M} 1416655$

Introduction. The mitral valve is one of the four cardiac valves. It lies between the left atrium and the left ventricle, and, different from the other three valves, it is basically made of two flaps. During diastole, the valve opens, allowing blood to flow from the atrium into the ventricle, while, during systole, the valve closes. The valve opens and closes because of pressure difference: it opens when the pressure is greater in the atrium than in the ventricle and closes vice versa [11].

The mitral valve has two leaflets: the anteromedial one and the posterolateral one (see Figure 0.1). The outer boundary of the mitral valve is attached to a fibrous ring known as the mitral annulus. The anteromedial leaflet covers approximately one-third of the valve perimeter, while the remaining two-thirds are covered by the posterolateral leaflet [7]. Valve tissue consists of a fibrous network, mainly collagen and elastin, saturated with a fluid that is mostly water [19]. The valve leaflets are prevented from prolapsing into the atrium by the action of the chordae tendineae. These are inelastic tendons, much like parachute wires, attached, at one end, to the inferior surface of the valve leaflets and, at the other end, to the papillary muscles, which are part of the ventricular wall [13]. When the left ventricle contracts, the pressure in the ventricle forces the valve to close, while these tendons stop the leaflets in the correct position, coapting together, and prevent them from opening in the wrong direction. Each chord has a different thickness: the thinnest ones are attached

\footnotetext{
*Received by the editors April 30, 2021; accepted for publication (in revised form) August 27, 2021; published electronically January 6, 2022.

https://doi.org/10.1137/21M1416655

Funding: The authors acknowledge the support from the Italian Ministry of University and Research through the grant N.2017KL4EF3 (Mathematics of active materials: From mechanobiology to smart devices). The first and fourth authors acknowledge the partial support of the MIUR grant Dipartimenti di Eccellenza 2018-2022 (E11G18000350001).

${ }^{\dagger}$ DISMA, Politecnico di Torino, Corso Duca degli Abruzzi 24, 10129 Torino, Italy (davide. ambrosi@polito.it, marta.zoppello@polito.it).

${ }^{\ddagger}$ Pediatric Cardiac Surgery Department, Ospedale Infantile Regina Margherita, Piazza Polonia 94, 10126 Torino, Italy (deorsola@libero.it).

$\S$ Dipartimento di Matematica, Politecnico di Milano, Piazza Leonardo da Vinci 32, 20133 Milano, Italy (stefano.turzi@polimi.it).
} 

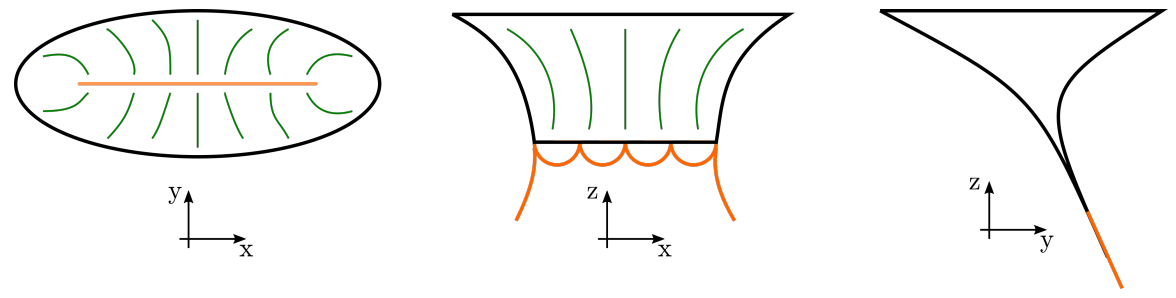

FIG. 0.1. Plane projections of a pictorial representation of the mitral valve: top view (left) and side views (right). The leaflets are drawn in blue; the embedded collagen fibers are green, while the chordae tendineae are orange. Our model is set up in the $(y, z)$ plane.

to the nonadhering part of the leaflet, whereas the thickest ones (that play the major structural role) are attached near to the contact point.

In the embryo the mitral valve originates from the so-called endocardial cushions, which is a thickening of the ventricular wall located immediately beneath the valve annulus [9]. During the initial phase, within these "cushions," a space is progressively created, which separates a layer of cells from the cardiac wall. Later, some "fenestrations" develop in the inferior area of this layer of cells, and the endocardial cushion space is thus put in communication with the ventricle. This allows the blood to enter the space and, with its flow and pressure, to force the endocardial cell layer to grow and to differentiate: the superior part of the layer generates the leaflets, while the inferior part (the fenestrated one) creates the chordal system. This process continues until the leaflets get in touch, and the valve assumes it final configuration. Hydrodynamic forces are essential to this process [14].

The biomechanical engineering literature devoted to modeling the mechanics of the mitral valve is huge [8] and is largely centered in developing three-dimensional finite element models that can represent the time-dependent solid-fluid dynamics interaction of the valve with the leaflets. The approach of this work is different: we develop a reduced order model that is able to capture essential geometrical features of the mitral valve that are apparent at ecocardiography and can support a surgeon in designing his practice.

A relevant work inspired by the minimalistic approach is due to Arts et al. [1]. They study the mitral valve as a membrane, exploiting the Laplace law: without detailing constitutive material laws and using differential geometry arguments, they derive some analytical relations that exist between apparent geometrical characteristics of the valve at systole (length of the nonattached flaps, volume of the chamber) and the tension applied by the cordae tendineae.

In this work we consider a section of the heart along the base-to-apex direction (see Figure 0.1(right)). During the morphogenesis of the valve, the flaps are mathematically represented as two noninteracting rods, with one endpoint attached to a wire that constraints its kinematics. In the mature mitral valve, the leaflets are represented as rods of different lengths that at systole partially overlap each other. The rods have an edge fixed in the plane (on the annulus); they are inextensible and subject to a pressure difference. We focus on the end of the systolic phase when the rod bending produced by the pressure difference is balanced by the tension of the chordae tendineae and by the interaction between the flaps in contact.

The mathematical derivation of the model is carried out for the more complex system, i.e., the mature mitral valve with partially overlapping leaflets; the mathematical model for the growing no-contact flap follows under some simplifications. We 
ideally split every rod in its attached and unattached portion, and the equilibrium equations are obtained exploiting the principle of virtual work, written for rods under the action of the pressure force per unit length. After the first variation of the energy functional, we obtain a system of three differential equations, supplemented by suitable boundary conditions and two nonlinear algebraic equations that define the curvilinear coordinates of the attachment point [16].

An asymptotic analysis points out the existence of boundary layers in the pressure regime of interest, and we argue that a smart choice of the boundary conditions can suppress the large tensional gradients that in general occur at a boundary. Numerical integrations are performed for physiological values of the parameters and confirm in an excellent way the approximate solutions obtained by asymptotic analysis. The computed angles between the leaflets and the plane of the annulus are compared with the ones measured by anatomical studies and ultrasound imaging.

1. The two rod system. In this section we obtain the equations for two rods in contact that represent a bare-bones mechanical model of a mitral valve. The derivation follows a variational approach [16]. The simpler system, i.e., the leaflets at the embryonic stage, are represented by a single growing leaflet without contact, and their mathematical model follows from the general one under simplifying assumptions.

1.1. Kinematics. We consider a mechanical system composed by two inextensible rods that are in contact for a portion of their length. We therefore conveniently introduce three curves in the plane [12]: the first two describe the position of nonattached rods, subject to pressure difference; the latter accounts for the partially attached configuration

$$
\mathbf{r}_{i}\left(s_{i}\right):\left[0, \bar{s}_{i}\right] \rightarrow \mathbb{R}^{2}, \quad i=1,2,3,
$$

where $\mathbf{r}_{i}\left(s_{i}\right)=\left(x\left(s_{i}\right), y\left(s_{i}\right)\right)$ and in the mutual attachment point (the interface)

$$
\mathbf{r}_{1}\left(\bar{s}_{1}\right)=\mathbf{r}_{2}\left(\bar{s}_{2}\right)=\mathbf{r}_{3}\left(\bar{s}_{3}\right) .
$$

The two leaflets have a portion of the boundary on the annulus so that rods $\mathbf{r}_{1}$ and $\mathbf{r}_{2}$ have a fixed endpoint

$$
\mathbf{r}_{1}(0)=\mathbf{r}_{1}^{0}, \quad \mathbf{r}_{2}(0)=\mathbf{r}_{2}^{0}
$$

while the endpoint of the attached portions of the rods $\mathbf{r}_{3}(0)$ is free (see Figure 1.1).

A unit tangent vector $\mathbf{t}_{i}=\left(\cos \left(\theta_{i}\right), \sin \left(\theta_{i}\right)\right)$ is uniquely defined in every point of a curve, where $\theta_{i}\left(s_{i}\right)$ is the angle between the tangent vector and the $x$-axis. Normal unit vectors are defined as $\mathbf{n}_{i}=\left(\sin \left(\theta_{i}\right),-\cos \left(\theta_{i}\right)\right)$ so that $\mathbf{t}_{i}^{\prime}=-\mathbf{n}_{i} \theta_{i}^{\prime}$. In the attachment point the tangent vector is smooth:

$$
\theta_{1}\left(\bar{s}_{1}\right)=\theta_{2}\left(\bar{s}_{2}\right)=\theta_{3}\left(\bar{s}_{3}\right)+\pi .
$$

We adopt arc-length parametrizations so that

$$
\bar{s}_{1}+\bar{s}_{3}=\ell_{1}, \quad \bar{s}_{2}+\bar{s}_{3}=\ell_{2},
$$

where $\ell_{1}$ and $\ell_{2}$ are the rods' lengths. We notice that $\ell_{1}$ and $\ell_{2}$ are not the physical length of the flaps, but the length measured from the points $\mathbf{r}_{i}(0), i=1,2$, to the attachment point $A$; the length of the remaining portion of the valve in the ventricle plays no mechanical role, and it is immaterial for the present model. 


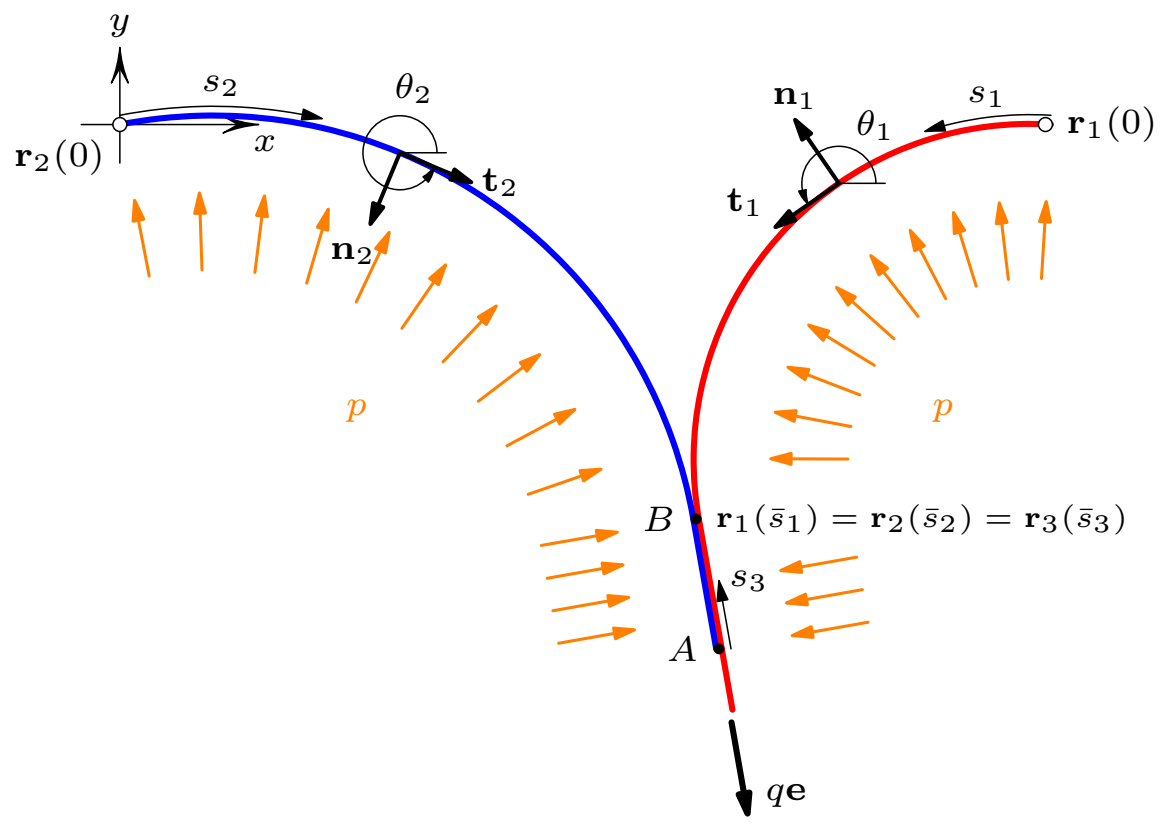

FIG. 1.1. The mitral valve is mechanically represented as a system of three rods: two of them $\left(\mathbf{r}_{1}\left(s_{1}\right), \mathbf{r}_{2}\left(s_{2}\right)\right)$ have a fixed endpoint; the latter one $\left(\mathbf{r}_{3}\left(s_{3}\right)\right)$ represents the contact region where the flaps attatch to each other. The leaflets partially lay one over the other, starting from the common point $\mathbf{r}_{1}\left(\bar{s}_{1}\right)=\mathbf{r}_{2}\left(\bar{s}_{2}\right)=\mathbf{r}\left(\bar{s}_{3}\right)$. The pressure difference $p$ along the rods is represented with orange arrows. A wire connects the endpoint of the lower rod with a fixed point, and its action is dynamically represented by the force qe.

Moreover the following relations hold:

$$
\mathbf{n}_{1}\left(\bar{s}_{1}\right)=\mathbf{n}_{2}\left(\bar{s}_{2}\right)=-\mathbf{n}_{3}\left(\bar{s}_{3}\right),
$$

while

$$
\mathbf{t}_{1}\left(\bar{s}_{1}\right)=\mathbf{t}_{2}\left(\bar{s}_{2}\right)=-\mathbf{t}_{3}\left(\bar{s}_{3}\right) .
$$

Remark 1. In real physiology, the first contact point between the leaflets of the mitral valve during closure is dictated by time-dependent dynamics: the blood pressure pushes the open flaps from the cardiac wall to the annulus plane, and their first contact occurs in some specific point, as driven by the morphology, by the blood flow and by its interaction with the deformable leaflet surfaces. As the surface of the flaps are rough, they do not slide over each other after first contact, and such a point remains fixed.

In our static structural model, we are not able to solve the dynamics that determines the early contact point, which is therefore here a datum of the curvilinear coordinates of the earliest contact point $\ell_{1}$ and $\ell_{2}$ (point $\mathrm{A}$ in Figure 1.1). The curvilinear coordinate of the triple point (point B in Figure 1.1) is the length of the contact region, and it is instead an unknown of the problem. In the final section we shall consider different values of $\ell_{1}$ and $\ell_{2}$ corresponding to different positions of the early attachment point.

The no-slip condition that characterizes the leaflet surfaces here yields a "three rod" model, in contrast with the "four rod" model that is able to account for the 
perfect slip condition: the flaps can freely slide over each other, and the early contact point is an immaterial information, as discussed, for instance, in [16].

1.2. Equilibrium equations. The inextensible rods store elastic energy only because of bending:

$$
w_{i}\left(\theta_{i}^{\prime}, \bar{s}_{i}\right)=\int_{0}^{\bar{s}_{i}} \frac{k_{i}(s)}{2}\left(\theta_{i}^{\prime}\right)^{2} d s_{i}
$$

where $k_{i}(s), i=1,2,3$, are bending moduli which possibly vary along each rod due to their different thickness and $k_{1}=k_{2}$ and $k_{3}=4 k_{1}+4 k_{2}$, as obtained with the layered structure of the physical leaflets.

We add the inextensibility constraint to the energy, and we get

$$
W\left(\mathbf{r}_{i}, \theta_{i}, \mathbf{N}_{i}, \bar{s}_{i}\right)=\sum_{i=1}^{3}\left(\int_{0}^{\bar{s}_{i}} \frac{k_{i}(s)}{2}\left(\theta_{i}^{\prime}\right)^{2} d s_{i}-\int_{0}^{\bar{s}_{i}} \mathbf{N}_{i} \cdot\left(\mathbf{r}_{i}^{\prime}-\mathbf{t}_{i}\right) d s_{i}\right),
$$

where $\mathbf{N}_{i}\left(s_{i}\right)=\left(N_{i}^{x}\left(s_{i}\right), N_{i}^{y}\left(s_{i}\right)\right)$ is the (unknown) reaction force in the $i$ th rod.

In our system a pressure difference $p$ applies to the rods that separate the two cardiac chambers, i.e., $\mathbf{r}_{1}$ and $\mathbf{r}_{2}$. One may notice that the force per unit length $p \mathbf{n}_{i}$ is neither conservative nor dissipative, so we use the nonconservative version of the principle of virtual works, $\delta W=\delta L$, where $\delta L$ is the work exerted by the nonconservative active forces.

Taking into account all the possible variations, including the contact point, the principle reads

$$
\begin{array}{r}
\sum_{i=1}^{3}\left(\int_{0}^{\bar{s}_{i}} k_{i} \theta_{i}^{\prime} \delta \theta_{i}^{\prime} d s_{i}-\int_{0}^{\bar{s}_{i}} \delta \mathbf{N}_{i} \cdot\left(\mathbf{r}_{i}^{\prime}-\mathbf{t}_{i}\right) d s_{i}-\int_{0}^{\bar{s}_{i}} \mathbf{N}_{i} \cdot\left(\delta \mathbf{r}_{i}^{\prime}+\mathbf{n}_{i} \delta \theta_{i}\right)\right) d s_{i} \\
+\sum_{i=1}^{3}\left[k_{i}\left(\theta_{i}^{\prime}\right)^{2}-\mathbf{N}_{i} \cdot\left(\mathbf{r}_{i}^{\prime}-\mathbf{t}_{i}\right)\right]_{\bar{s}_{i}} \delta \bar{s}_{i} \\
=\int_{0}^{\bar{s}_{1}} p \mathbf{n}_{1} \cdot \delta \mathbf{r}_{1} d s_{1}-\int_{0}^{\bar{s}_{2}} p \mathbf{n}_{2} \cdot \delta \mathbf{r}_{2} d s_{2} .
\end{array}
$$

After integration by parts we get

$$
\begin{array}{r}
\sum_{i=1}^{3}\left(-\int_{0}^{\bar{s}_{i}}\left(k_{i} \theta_{i}^{\prime}\right)^{\prime} \delta \theta_{i} d s_{i}+\left[k_{i} \theta_{i}^{\prime} \delta \theta_{i}\right]_{0}^{\bar{s}_{i}}-\int_{0}^{\bar{s}_{i}} \delta \mathbf{N}_{i} \cdot\left(\mathbf{r}_{i}^{\prime}-\mathbf{t}_{i}\right) d s_{i}+\int_{0}^{\bar{s}_{i}} \mathbf{N}_{i}^{\prime} \cdot \delta \mathbf{r}_{i}\left(\bar{s}_{i}\right) d s_{i}\right. \\
\left.\left.-\left[\mathbf{N}_{i} \cdot \delta \mathbf{r}_{i}\right]_{0}^{\bar{s}_{i}}-\int_{0}^{\bar{s}_{i}} \mathbf{N}_{i} \cdot \mathbf{n}_{i} \delta \theta_{i} d s_{i}\right)+\sum_{i=1}^{3}\left[\frac{k_{i}}{2}\left(\theta_{i}^{\prime}\right)^{2}-\mathbf{N}_{i} \cdot \mathbf{r}_{i}^{\prime}-\mathbf{t}_{i}\right)\right]_{\bar{s}_{i}} \delta \bar{s}_{i} \\
=\int_{0}^{\bar{s}_{1}} p \mathbf{n}_{1} \cdot \delta \mathbf{r}_{1} d s_{1}-\int_{0}^{\bar{s}_{2}} p \mathbf{n}_{2} \cdot \delta \mathbf{r}_{2} d s_{2} .
\end{array}
$$

The Euler-Lagrange equations of the system (1.11) are obtained by the independent variation of the fields of the energy functional (1.9): positions, angles, reactions, and the curvilinear coordinates $\bar{s}_{i}$. However, the variations $\delta \mathbf{r}_{i}\left(\bar{s}_{i}\right)$ and $\delta \theta_{i}\left(\bar{s}_{i}\right)$, appearing in (1.11) after integration by parts, contain contributions to the variations of $\bar{s}_{i}$. 
To decouple the increments we observe that the total variation of $\theta_{i}$, say $\delta \bar{\theta}_{i}$, up to first order is composed by two terms,

$$
\delta \bar{\theta}_{i}=\left(\theta_{i}\left(\bar{s}_{i}+\delta \bar{s}_{i}\right)+\delta \theta_{i}\left(\bar{s}_{i}+\delta \bar{s}_{i}\right)\right)-\theta\left(\bar{s}_{i}\right)=\theta^{\prime}\left(\bar{s}_{i}\right) \delta \bar{s}_{i}+\delta \theta_{i}\left(\bar{s}_{i}\right)
$$

and, analogously,

$$
\delta \overline{\mathbf{r}}_{i}=\mathbf{r}^{\prime}\left(\bar{s}_{i}\right) \delta \bar{s}_{i}+\delta \mathbf{r}_{i}\left(\bar{s}_{i}\right)
$$

We now take $\delta \bar{s}_{i}, \delta \bar{\theta}_{i}, \delta \overline{\mathbf{r}}_{i}$ as independent. Moreover, from the conditions (1.2) and (1.5) the following relations hold:

$$
\delta \mathbf{r}_{1}\left(\bar{s}_{1}\right)=\delta \mathbf{r}_{2}\left(\bar{s}_{2}\right)=\delta \mathbf{r}_{3}\left(\bar{s}_{3}\right), \quad \delta \bar{s}_{3}=-\delta \bar{s}_{1}, \quad \delta \bar{s}_{3}=-\delta \bar{s}_{2}, \quad \delta \bar{s}_{1}=\delta \bar{s}_{2} .
$$

Using (1.12) and (1.13) to represent $\delta \theta_{i}\left(\bar{s}_{i}\right)$ and $\delta \mathbf{r}_{i}\left(\bar{s}_{i}\right)$ and the relations (1.5), (1.11) rewrites

$$
\begin{array}{r}
\sum_{i=1}^{3}\left(-\int_{0}^{\bar{s}_{i}}\left(k_{i} \theta_{i}^{\prime}\right)^{\prime} \delta \theta_{i} d s_{i}+k_{i} \theta_{i}^{\prime}\left(\bar{s}_{i}\right) \delta \bar{\theta}_{i}-k_{i} \theta_{i}^{\prime}(0) \delta \theta_{i}(0)\right) \\
+\sum_{i=1}^{3}\left(-\int_{0}^{\bar{s}_{i}} \delta \mathbf{N}_{i} \cdot\left(\mathbf{r}_{i}^{\prime}-\mathbf{t}_{i}\right) d s_{i}+\int_{0}^{\bar{s}_{i}} \mathbf{N}_{i}^{\prime} \cdot \delta \mathbf{r}_{i} d s_{i}-\mathbf{N}_{i}\left(\bar{s}_{i}\right) \cdot \delta \overline{\mathbf{r}}_{i}\right. \\
\left.+\mathbf{N}_{i}(0) \cdot \delta \mathbf{r}_{i}(0)-\int_{0}^{\bar{s}_{i}} \mathbf{N}_{i} \cdot \mathbf{n}_{i} \delta \theta_{i} d s_{i}\right) \\
+\sum_{i=1}^{3}\left[\frac{k_{i}}{2}\left(\theta_{i}^{\prime}\right)^{2}-k_{i}\left(\theta_{i}^{\prime}\right)^{2}-\mathbf{N}_{i} \cdot\left(\mathbf{r}_{i}^{\prime}-\mathbf{t}_{i}\right)+\mathbf{N}_{i} \cdot \mathbf{r}_{i}^{\prime}\right]_{\bar{s}_{i}} \delta \bar{s}_{i} \\
=\int_{0}^{\bar{s}_{1}} p \mathbf{n}_{1} \cdot \delta \mathbf{r}_{1} d s_{1}-\int_{0}^{\bar{s}_{2}} p \mathbf{n}_{2} \cdot \delta \mathbf{r}_{2} d s_{2} .
\end{array}
$$

Now all independent variations can be carried out so that the following system of differential equations is obtained:

$$
\begin{aligned}
& \left(k_{i} \theta_{i}^{\prime}\right)^{\prime}+\mathbf{N}_{i} \cdot \mathbf{n}_{i}=0, \quad i=1,2,3, \\
& \mathbf{N}_{1}^{\prime}=p \mathbf{n}_{1}, \quad \mathbf{N}_{2}^{\prime}=-p \mathbf{n}_{2}, \\
& \mathbf{N}_{3}^{\prime}=0, \\
& \mathbf{r}_{i}^{\prime}=\mathbf{t}_{i}, \quad i=1,2,3 .
\end{aligned}
$$

The chordae tendineae are fixed on one side at the endpoint of rod 3, and on the other side they are attached to the papillary muscles, whose position is here denoted by $\mathbf{r}^{P}$. On the basis of the assumptions (1.2) and (1.3), the system of equations is supplemented by the boundary conditions

$$
\begin{array}{r}
\mathbf{r}_{1}(0)=\mathbf{r}_{1}^{0}, \quad \mathbf{r}_{2}(0)=\mathbf{r}_{2}^{0}, \quad \mathbf{N}_{3}(0)=q \mathbf{e}, \\
\theta_{i}^{\prime}(0)=0, \quad i=1,2,3,
\end{array}
$$

where $\mathbf{e}:=\frac{\mathbf{r}^{P}-\mathbf{r}_{3}(0)}{\left\|\mathbf{r}^{P}-\mathbf{r}_{3}(0)\right\|}$ denotes the direction of the force of intensity $q$ exerted by the chordae tendineae. 
Remark 2. The last boundary condition in (1.17a) assumes that the tension of the chordae tendineae $q$ is known. Dually, one could assume that a constraint applies: a chorda tendinea of given length connects the endpoint of the leaflet and a fixed point in the plane. Our approach simplifies the associated numerical problem. The two strategies are equivalent from a theoretical standpoint: in principle one can span the space of possible values of $q$ and then a posteriori identify the value of the parameter and the corresponding solution associated to a specific length of the chorda and a specific fixation point.

Remark 3 . The boundary condition $(1.17 \mathrm{~b})$ has a precise mechanical meaning: it assumes no torque at the boundary so that the leaflet can freely rotate around the fixed point. Another possible assumption could be to fix the angle $\theta=\bar{\theta}$ at the boundary, like in a wedged beam, or even to assume that a torsional spring applies, yielding a Robin-type boundary condition $\theta^{\prime}+\chi(\theta-\bar{\theta})=0$. As the leaflet during the cardiac cycle takes a range of different configurations, it is not obvious to discern the physiologically correct boundary condition on the basis of ecocardiographic observations. Notwithstanding such a difficulty, in section 2 we show that almost all boundary conditions generate, in the regime of interest for cardiac applications, a tensional boundary layer that can be avoided adopting a specific "optimal" choice.

In the triple point (point B in Figure1.1), (1.2) and (1.4) provide continuity of position and angles

$$
\begin{array}{r}
\mathbf{r}_{1}\left(\bar{s}_{1}\right)=\mathbf{r}_{2}\left(\bar{s}_{2}\right)=\mathbf{r}_{3}\left(\bar{s}_{3}\right), \\
\theta_{1}\left(\bar{s}_{1}\right)=\theta_{2}\left(\bar{s}_{2}\right)=\theta_{3}\left(\bar{s}_{3}\right)+\pi
\end{array}
$$

and are complemented by the balance of forces and torques derived from the boundary terms of (1.15):

$$
\begin{aligned}
\mathbf{N}_{1}\left(\bar{s}_{1}\right)+\mathbf{N}_{2}\left(\bar{s}_{2}\right)+\mathbf{N}_{3}\left(\bar{s}_{3}\right) & =0, \\
k_{1}\left(\bar{s}_{1}\right) \theta_{1}^{\prime}\left(\bar{s}_{1}\right)+k_{2}\left(\bar{s}_{2}\right) \theta_{2}^{\prime}\left(\bar{s}_{2}\right)+k_{3}\left(\bar{s}_{3}\right) \theta_{3}^{\prime}\left(\bar{s}_{3}\right) & =0 .
\end{aligned}
$$

The extra terms due to the total variations of the fields in the unknown contact point, appearing in (1.15) in square brackets, can be understood as a continuity of the Legendre transform of the strain energy (the Hamiltonian) or, in other contexts, as the continuity of the Eshelby stress. Using (1.5) and (1.19) we eventually find the condition that defines the curvilinear coordinates $\bar{s}_{1}$ and $\bar{s}_{2}$ of the interface point

$$
\left[-\frac{k_{1}}{2}\left(\theta_{1}^{\prime}\right)^{2}+\mathbf{N}_{1} \cdot \mathbf{t}_{1}\right]_{s=\bar{s}_{1}}+\left[-\frac{k_{2}}{2}\left(\theta_{2}^{\prime}\right)^{2}+\mathbf{N}_{2} \cdot \mathbf{t}_{2}\right]_{s=\bar{s}_{2}}\left[-\frac{k_{3}}{2}\left(\theta_{3}^{\prime}\right)^{2}+\mathbf{N}_{3} \cdot \mathbf{t}_{3}\right]_{s=\bar{s}_{3}}=0
$$

where relations (1.7), (1.14), and (1.16c) have been used. Using the relation existing between normal vectors in the triple point (1.7) and the relation between reaction forces (1.19), the last condition (1.20) simplifies as follows:

$$
-\left.\frac{k_{1}}{2}\left(\theta_{1}^{\prime}\right)^{2}\right|_{s=\bar{s}_{1}}-\left.\frac{k_{2}}{2}\left(\theta_{2}^{\prime}\right)^{2}\right|_{s=\bar{s}_{2}}+\left.\frac{k_{3}}{2}\left(\theta_{3}^{\prime}\right)^{2}\right|_{s=\bar{s}_{3}}=0
$$

The statement of the differential problem is well posed: there are 18 unknown fields, i.e., $\theta_{i}, \theta_{i}^{\prime}, N_{i}^{x}, N_{i}^{y}, x_{i}$, and $y_{i}$ for $i=1,2,3$, plus $\bar{s}_{3}$. The system (1.16) contains three second order scalar differential equations and six vectorial first order equations, requiring altogether 18 boundary conditions plus an algebraic equation provided by (1.17), (1.18), (1.19), and (1.21). The differential system is fully coupled: despite the 
fact that (1.16a) and (1.16b) apparently do not involve the position, their boundary conditions do.

Remark 4. The system of equations (1.16)-(1.21) can be integrated immediately for rod 3. From (1.16c), using the boundary condition (1.17a), we get

$$
\mathbf{N}_{3}(s)=q \mathbf{e} .
$$

Equation (1.16a) multiplied times $\theta_{3}^{\prime}$ can be integrated thus obtaining

$$
\frac{k_{3}(s)}{2}\left(\theta_{3}^{\prime}(s)\right)^{2}=-\int_{0}^{s} \mathbf{N}_{3} \cdot \mathbf{n}_{3} \theta_{3}^{\prime} d s=q \mathbf{e} \cdot\left(\mathbf{t}_{3}(s)-\mathbf{t}_{3}(0)\right)
$$

that has solution $\theta_{3}(s)=\theta_{3}(0)=\theta_{3}\left(\bar{s}_{3}\right)$ : the rod 3 is straight, with an inclination $\theta_{3}$ to be determined.

2. Asymptotic analysis. In this section we carry out an asymptotic analysis of the nonlinear problem of a single leaflet subjected to the blood pressure and to the boundary tension produced by the chordae tendineae under the assumption that they detach from the tip parallel to the leaflet. Enforcing the large pressure regime that characterizes the cardiac flow (see section 3.1), we exploit the existence of a small parameter, and we demonstrate that tensional boundary layers exist for almost every boundary condition. The perturbation results will be compared with fully nonlinear numerical simulations in section 3.

Consider a single leaflet with constant bending modulus $k$. The equations of the mechanical system follow from (1.16) with obvious simplification and notation:

$$
\begin{aligned}
k \theta^{\prime \prime}+\mathbf{N} \cdot \mathbf{n} & =0, \\
\mathbf{N}^{\prime}+p \mathbf{n} & =0 .
\end{aligned}
$$

Representing the tension in its normal and tangential components to the beam, $\mathbf{N}=$ $N_{n} \mathbf{n}+N_{t} \mathbf{t}$, and using $\mathbf{N}^{\prime}=N_{t}^{\prime} \mathbf{t}-N_{t} \theta^{\prime} \mathbf{n}+N_{n}^{\prime} \mathbf{n}+N_{n} \theta^{\prime} \mathbf{t}$, (2.1) rewrites

$$
\begin{aligned}
k \theta^{\prime \prime}+N_{n} & =0, \\
N_{t}^{\prime}+N_{n} \theta^{\prime} & =0, \\
N_{n}^{\prime}-N_{t} \theta^{\prime}+p & =0 .
\end{aligned}
$$

Multiplying (2.2) by $\theta^{\prime}$ we get

$$
\begin{aligned}
k \theta^{\prime \prime} \theta^{\prime}+N_{n} \theta^{\prime} & =\left(\frac{1}{2} k \theta^{\prime 2}\right)^{\prime}+\mathbf{N} \cdot \theta^{\prime} \mathbf{n}=\left(\frac{1}{2} k \theta^{\prime 2}\right)^{\prime}-\mathbf{N} \cdot \mathbf{t}^{\prime} \\
& =\left(\frac{1}{2} k \theta^{\prime 2}\right)^{\prime}-(\mathbf{N} \cdot \mathbf{t})^{\prime}+\mathbf{N}^{\prime} \cdot \mathbf{t}=\left(\frac{1}{2} k \theta^{\prime 2}\right)^{\prime}-(\mathbf{N} \cdot \mathbf{t})^{\prime}=0
\end{aligned}
$$

so that

$$
N_{t}=\frac{1}{2} k \theta^{\prime 2}+c
$$

where $c$ is a constant to be determined by the boundary conditions. It is natural to assume that the no external bending moment applies at $s=0$ and $s=L$ so that we posit

$$
\theta^{\prime}(0)=0, \quad \theta^{\prime}(L)=0
$$

Copyright $@$ by SIAM. Unauthorized reproduction of this article is prohibited. 
Furthermore, we introduce the angle $\Omega$ between the direction of the beam at the boundary $\mathbf{e}$ and the tangent $\mathbf{t}$ so that $\mathbf{e} \cdot \mathbf{t}=\cos \Omega, \mathbf{e} \cdot \mathbf{n}=\sin \Omega$. Hence,

$$
N_{t}(L)=-q \cos \Omega, \quad N_{n}(L)=-q \sin \Omega .
$$

Using the boundary conditions (2.7) and (2.8) in $s=L$, we obtain $c=-q \cos \Omega$. After derivation of $(2.2),(2.4)$ rewrites as a decoupled second order differential equation for the curvature $\theta^{\prime}(s)$, involving the unknown angle $\Omega$ :

$$
k \theta^{\prime \prime \prime}+\frac{1}{2} k \theta^{\prime 3}-(q \cos \Omega) \theta^{\prime}-p=0 .
$$

We now recast (2.9) in dimensionless form in terms of the scaled variable $\sigma=s / L$ and the function $z(\sigma)=\frac{\partial \theta}{\partial \sigma}(\sigma)$. Hence, (2.9) rewrites as

$$
z^{\prime \prime}+\frac{1}{2} z^{3}-\frac{q L^{2}}{k} z \cos \Omega-\frac{p L^{3}}{k}=0,
$$

where now $(\cdot)^{\prime}$ stands for the derivative with respect the dimensionless variable $\sigma$. We are interested in studying this equation in the limit $\hat{\alpha}=p L^{3} / k \rightarrow+\infty$, which corresponds to the high pressure regime characterizing the physiological state of the mitral valve (see the discussion in section 3.1). In this limit, the last term must be balanced by the third one so that $p L^{3} / k$ and $q L^{2} / k$ must be of the same order. Hence, we define $\eta=q / p L$ and $\epsilon=1 / \hat{\alpha}=k /\left(p L^{3}\right)$, and the dimensionless problem reads

$$
\left\{\begin{array}{l}
\epsilon z^{\prime \prime}+\frac{1}{2} \epsilon z^{3}-(\eta \cos \Omega) z-1=0 \\
z(0)=0 \\
z(L)=0
\end{array}\right.
$$

The solution away from the boundaries (the outer solution) is obtained, to zeroth order, by setting $\epsilon=0$, and it is therefore $z_{\text {out }}=-1 /(\eta \cos \Omega)$. The function $z_{\text {out }}$ does not satisfy the boundary conditions; therefore there must exist a solution defined in a boundary layer (the inner solution) that smoothly connects $z_{\text {out }}$ with the boundary values. The uniform solution can be found by the method of matched asymptotic expansions [2] described below.

- At the boundary $\sigma=0$, we set $X=\sigma / \delta(\epsilon)$, with $\delta \ll 1$ as $\epsilon \rightarrow 0$. The layer thickness $\delta$ is yet to be determined. A magnification of the solution in the boundary layer is obtained by rescaling $z(\sigma)=\left.Z(X)\right|_{X=\sigma / \delta}$. Equation (2.11) becomes

$$
\frac{\epsilon}{\delta^{2}} Z^{\prime \prime}+\frac{1}{2} \epsilon Z^{3}-(\eta \cos \Omega) Z-1=0 .
$$

A dominant balance argument shows that the distinguished limit is $\epsilon / \delta^{2}=1$, which yields $\delta(\epsilon)=\sqrt{\epsilon}$. We can now write the inner solution in the boundary layer in terms of such a small parameter $Z_{\text {inn, } 1}(X)=Z_{0}(X)+\sqrt{\epsilon} Z_{1}(X)+$ $O(\epsilon)$. The leading order problem is

$$
\left\{\begin{array}{l}
Z_{0}^{\prime \prime}-(\eta \cos \Omega) Z_{0}-1=0 \\
Z_{0}(0)=0
\end{array}\right.
$$

whose solution is $Z_{0}(X)=-\frac{1}{\eta \cos \Omega}\left(1-\mathrm{e}^{-X \sqrt{\eta \cos \Omega}}\right)$. We observe that the other possible solution, namely, $\mathrm{e}^{X \sqrt{\eta \cos \Omega}}$, must be discarded since it cannot match the outer solution (it diverges as $X \rightarrow \infty$ ). 
- At the boundary $\sigma=1$, we define the inner variable $X=(1-\sigma) / \delta$. Like in the previous case, (2.11) takes the form (2.12); thus the thickness of the boundary layer is again $\delta=\sqrt{\epsilon}$, and the solution $Z_{\mathrm{inn}, 2}(X)$ to leading order is again $Z_{\text {inn, }, 2}(X)=-\frac{1}{\eta \cos \Omega}\left(1-\mathrm{e}^{-X \sqrt{\eta \cos \Omega}}\right)$, with the only difference that the inner variable is now defined as $X=(1-\sigma) / \sqrt{\epsilon}$.

We notice that in both cases there are no integration constants because they have been ruled out by the boundedness of the inner solutions. However, matching to leading order is satisfied since $\lim _{X \rightarrow+\infty} Z_{0}(X)=\lim _{\sigma \rightarrow 0} z_{0}(\sigma)=-1 /(\eta \cos \Omega)$. Finally, the uniform solution is

$$
\begin{aligned}
z_{\mathrm{uni}}(\sigma) & =Z_{\text {inn }, 1}(\sigma)+z_{\text {out }}(\sigma)+Z_{\text {inn }, 2}(\sigma)-z_{\text {match }, 1}(\sigma)-z_{\text {match }, 2}(\sigma) \\
& =-\frac{1}{\eta \cos \Omega}\left(1-\mathrm{e}^{-\sigma \sqrt{\frac{\eta \cos \Omega}{\epsilon}}}\right)-\frac{1}{\eta \cos \Omega}\left(1-\mathrm{e}^{-(1-\sigma) \sqrt{\frac{\eta \cos \Omega}{\epsilon}}}\right)+\frac{1}{\eta \cos \Omega} \\
& =-\frac{1}{\eta \cos \Omega}\left(1-\mathrm{e}^{-\sigma \sqrt{\frac{\eta \cos \Omega}{\epsilon}}}-\mathrm{e}^{-(1-\sigma) \sqrt{\frac{\eta \cos \Omega}{\epsilon}}}\right) .
\end{aligned}
$$

Interestingly, the nonlinear term $\frac{1}{2} z^{3}$ does not affect the solution to leading order, but it only enters in the higher order approximations. In dimensional form, the curvature of the mitral leaflets is

$$
\theta^{\prime}(s)=\frac{\mathrm{d} \theta}{\mathrm{d} s}=\frac{1}{L} z_{\mathrm{uni}}(s / L)=-\frac{p}{q \cos \Omega}\left(1-\mathrm{e}^{-s \sqrt{q \cos \Omega / k}}-\mathrm{e}^{-(L-s) \sqrt{q \cos \Omega / k}}\right) .
$$

Finally, in order to find the angle $\Omega$, we use the remaining boundary condition, namely, $(2.8 \mathrm{~b})$, and $(2.2)$ evaluated in $s=L$ :

$$
k \theta^{\prime \prime}(L)=q \sin \Omega .
$$

We notice that the second order equation (2.2) provides the supplemental boundary condition for the third equation (2.9) that has been obtained by further derivation.

Now $\theta^{\prime \prime}(L)$ is obtained by differentiating (2.15) and setting $s=L$; in dimensionless form, after some algebra, (2.16) reads

$$
1-\mathrm{e}^{-\sqrt{\frac{\eta \cos \Omega}{\epsilon}}}=\eta \sin \Omega \sqrt{\frac{\eta \cos \Omega}{\epsilon}},
$$

which implicitly defines the angle $\Omega$. To leading order, when $\epsilon \rightarrow 0$, the exponential rapidly vanishes, and the left-hand side is finite. Therefore, it must be that $\Omega(\epsilon) \ll 1$ to balance the diverging term on the right-hand side of (2.17), and, to leading order, we find

$$
\Omega \sim \sqrt{\epsilon / \eta^{3}}
$$

this is a small angle as $\epsilon \rightarrow 0$ so that $\cos \Omega$ can be safely approximated to 1 . In physical terms, this means that the chordae tension is parallel to the leaflet free end, a rather natural condition in the high pressure regime we consider.

The condition $\cos \Omega \approx 1$ allows us to further simplify the curvature of the leaflet so that we can write

$$
\theta^{\prime}(s)=-\frac{p}{q}\left(1-\mathrm{e}^{-s \sqrt{q / k}}-\mathrm{e}^{-(L-s) \sqrt{q / k}}\right) .
$$


2.1. Boundary layers and boundary conditions. Summarizing, the asymptotic analysis tells us that in the regime of high pressure, the leaflet takes a constant curvature away from the boundary (a circular shape of radius $q / p$ ), and near its edges there are two boundary layers of thickness $\sqrt{k / q}$. While the perturbative analysis holds under assumption of small $\epsilon$ (small bending modulus versus pressure), the curvature taken by the rod is independent of the material properties and depends only on the ratio of the pressure versus the edge force (as defined in the parameter $\eta$ ). The outer solution is nothing but the Laplace law, and it coincides with the solution found by Arts et al. [1].

On the basis of the degrees of freedom in the enforcement of boundary conditions discussed in the Remark 3, we wonder if the boundary layers could be removed by a suitable choice. The answer is positive: if the boundary condition matches the outer constant solution $(z(0)=-1 / \eta$ or $z(1)=-1 / \eta)$, the inner region vanishes. This nice mathematical condition is, however, not meaningful from a mechanical point of view: by definition $z=\theta^{\prime}(\sigma)$, and imposing a fixed value for $\theta^{\prime}(0)$ means that a pointwise torque should apply at the boundaries, a condition far from physiological reality. On the other hand, we conjecture that a distinguished Dirichlet boundary condition on $\theta(0)$ (not on its derivative) could remove the boundary layer too. While this theoretical result is more involved to obtain analytically because of the entangled boundary conditions of the system of equations (2.1), we show by numerical simulations that our intuition is correct: there exists a boundary condition $\theta(0)=\bar{\theta}$ that drives $\theta^{\prime}(0)$ to the Laplace solution everywhere and makes the tensional boundary layer vanish.

\section{Numerical simulations.}

3.1. Nondimensional numbers and parameters. In this section we address the numerical integration of the nonlinear equations that model the equilibrium of a single leaflet and a closed mature mitral valve. Numerical results of the integration of the system of equations (2.1) ((1.16)-(1.20), respectively) are shown in a range of parameters of physiological interest.

A dimensional analysis of (1.16) reveals that there are four relevant nondimensional parameters of the full nonlinear system of equations:

$$
\alpha=P d^{4} / \bar{k}, \quad \beta=q / P d^{2}, \quad \gamma_{1}=\frac{\ell_{1}}{d}, \quad \gamma_{2}=\frac{\ell_{2}}{d},
$$

where $\bar{k}$ is a representative value of $k(s)$.

Typical physiological values of the parameters for an healthy patient are the systolic difference between chambers $P=120 \mathrm{~mm} \mathrm{Hg}$, the diameter of the mitral valve annulus $d=30 \mathrm{~mm}$ [7], and the bending modulus $\bar{k} \simeq 10-100 \mathrm{mN} \mathrm{mm}^{2}$ [4], thus yielding a typical value $\alpha \simeq 10^{5}-10^{6}$. Notice that the pressure $p$ of our bidimensional model (1.16b) is a force per unit length, while $P$ is a force per unit surface: in principle, the unknown $p$ should be read as the integral of $P$ along the third spatial dimension, which is not represented in this model.

The thickness of the leaflets varies along the curvilinear coordinate; this variation is immaterial for our rod model but reflects in the bending modulus $k(s)$ that depends quadratically on the thickness. We therefore smoothly modulate $k(s)$ around $\bar{k}$ from stiffer values near to the annulus to a softer modulus in the middle region for an order of magnitude.

The nondimensional parameter $\beta$ accounts for the balance of forces orthogonally to the rod and for the simulations is chosen to be $\beta \simeq 0.2$. In the same vein, the parameters $\gamma_{1}$ and $\gamma_{2}$ represent the ratio of the length of each rod from their fixed 
endpoints to the triple attachment point $A$ of Figure 1.1 (see Remark 1) to the distance $d$. From echocardiographic observations they typically range around $\gamma_{1} \simeq 0.8$ and $\gamma_{2} \simeq 0.98$. On the same echocardiographical basis, for numerical convenience we enforce that the unit vector e has a given direction, forming an angle $\frac{\pi}{18}$ with the vertical. In principle, if an information on $\mathbf{r}^{P}$ is provided, the orientation of $\mathbf{e}$ can be varied until the correct anchoring point is reproduced.

3.2. Single leaflet (primordial mitral valve). The single leaflet system studied in section 2 has not only an interest as a reduced system that can be more easily tackled by mathematical techniques but also has a biological relevance as a distinguished embryological subject per se. In the embryo the mitral valve originates from the so-called endocardial cushions, which is a thickening of the ventricular wall located immediately beneath the valve annulus [9]. During the initial phase, within these "cushions," a space is progressively created, which separates a layer of cells from the cardiac wall. Later, some "fenestrations" develop in the inferior area of this layer of cells, and the endocardial cushion space is thus put in communication with the ventricle. This allows the blood to enter the space and, with its flow and pressure, to force the endocardial cell layer to grow and to differentiate: the superior part of the layer generates the leaflets, while the inferior part (the fenestrated one) creates the chordal system. This process continues until the leaflets get in touch and the valve assumes its final configuration. Hydrodynamic forces are essential to this process [14].

In the framework of the mechanical model at hand, an immature mitral valve is therefore composed by two flaps that do not lean against each other; they only take a geometrical configuration produced by a balance of pressure and wire tension.
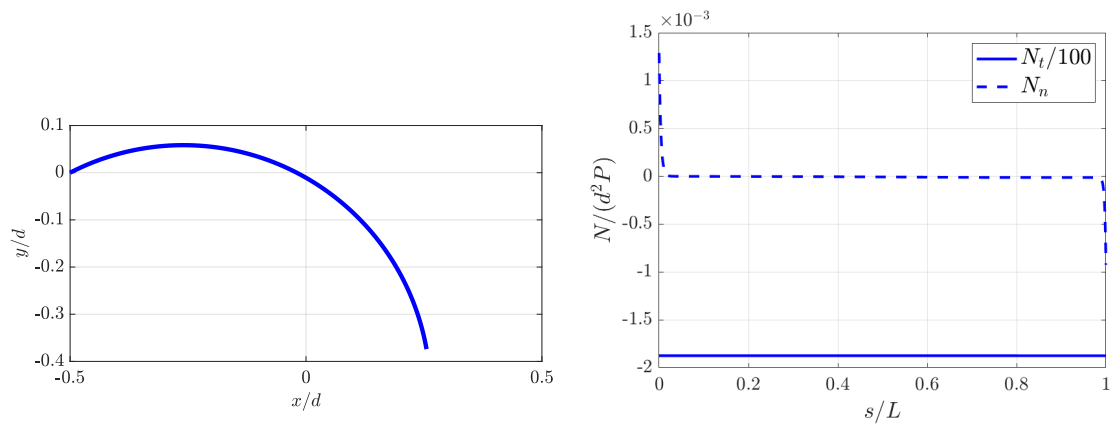

FIG. 3.1. Profile of a single leaflet of the mitral valve (left) for $\alpha=3 \cdot 10^{5}$ and $\beta=0.2$ : the shape is dictated by the balance of pressure and force produced by the chordae tendineae in the direction tangent to the flap without leaning on the other one. The tangent tension $N_{t}$ is constant; the normal tension in the leaflet $N_{n}$ is negligible.

The results of the numerical integration of (2.1) with boundary conditions (2.7) are reported in Figure 3.1, where a flap is supposed to be grown enough to be very close to touching the other one (not shown). The mechanical regime is defined by the nondimensional numbers $\alpha=3 \cdot 10^{5}$ and $\beta=0.2$. In such a regime, we can assume that the blood flow through the valve is very small and the pressure is not significantly different from the systolic peak. The numerical results correspond therefore to the same nondimensional data to be adopted for the healthy mature mitral valve in the next section. As expected, the shape of the flap is circular, the curvature radius being $\left|1 / \theta^{\prime}\right|=q / p$ (Figure 3.1(left)) 
In agreement with the asymptotic analysis carried out in section 1.2, there are boundary layers in the normal tension $N_{n}$ at both the endpoints of the flap (Figure 3.1(right)).

As shown in Figure 3.2, the dimensionless curvature $L \theta^{\prime}(s)$ that results after numerical integration of the full nonlinear system compares very well with the one obtained by the asymptotic expansion in section 2 (the uniform solution $z_{\text {unif }}$; see (2.19) multiplied by $L$ ).

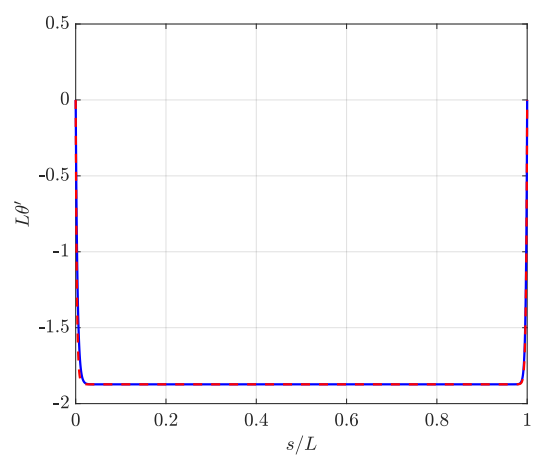

FIG. 3.2. Comparison between the nonlinear dimensionless curvature L $\theta^{\prime}$ (blue curve) obtained integrating the full nonlinear system, and the uniform solution obtained by asymptotic expansion (red dashed curve), as given in (2.19) multiplied by L.

On the basis of perturbative analysis, in section 2.1 we have conjectured that the boundary layer at the annulus can be eliminated by adopting a distinguished boundary condition in $s=0$ : instead of the hinge condition $\theta^{\prime}(0)=0$ we consider the leaflet as a wedge beam and thus impose the boundary condition $\theta(0)=\bar{\theta}$ for a suitable $\bar{\theta}$. As expected, the numerical results confirm our argument, for $\bar{\theta}=-27.32^{\circ}$ smoothly connects the circular outer solution with the boundary $\theta(0)$, thus suppressing the tensional boundary layer (see Figure 3.3).

Numerical results for $\beta=0.32$, corresponding to a stronger pulling force produced by the chordae tendineae, are reported in Figure 3.4 The rod bends more, and the optimal initial angle suppressing the left boundary layer is $\theta(0)=18.35^{\circ}$.

3.3. Mature mitral valve. Figure 3.5 shows the geometrical configuration of the leaflets of the mitral valve subject to a pressure difference obtained by numerical integration of (1.16)-(1.21). The mechanical regime is defined by the nondimensional numbers $\alpha=3 \cdot 10^{5}$ and $\gamma_{1}=0.8 \gamma_{2}=0.98$ in both cases. For $\beta=0.2$ (left) we have a physiologically correct placement of the leaflets, while for $\beta=0.1$ (right) the valve prolapses in the atrium because the tension of the chordae does not provide the correct balance to the pressure.

Figure 3.5(left) is in qualitative accordance with the shape of an healthy mitral valve as observed in echocardiography. The chordae form the expected angle of 10 degrees with the vertical axis. For a quantitative comparison, Table 3.1 reports the physiological relevant measures that are typically adopted in clinical practice as indicators of an healthy mitral valve:

- $\phi_{1}, \phi_{2}$ : positive angles between the leaflets and the plane of the annulus, formally $\phi_{1}:=\theta_{1}(0)-\pi$ and $\phi_{2}:=2 \pi-\theta_{2}(0)$;

- $h$ : distance between the flaps attachment point and the annular plane;

- $s_{1} / d, s_{2} / d$ : ratio between the length of non adhering leaflets and the annulus diameter. 

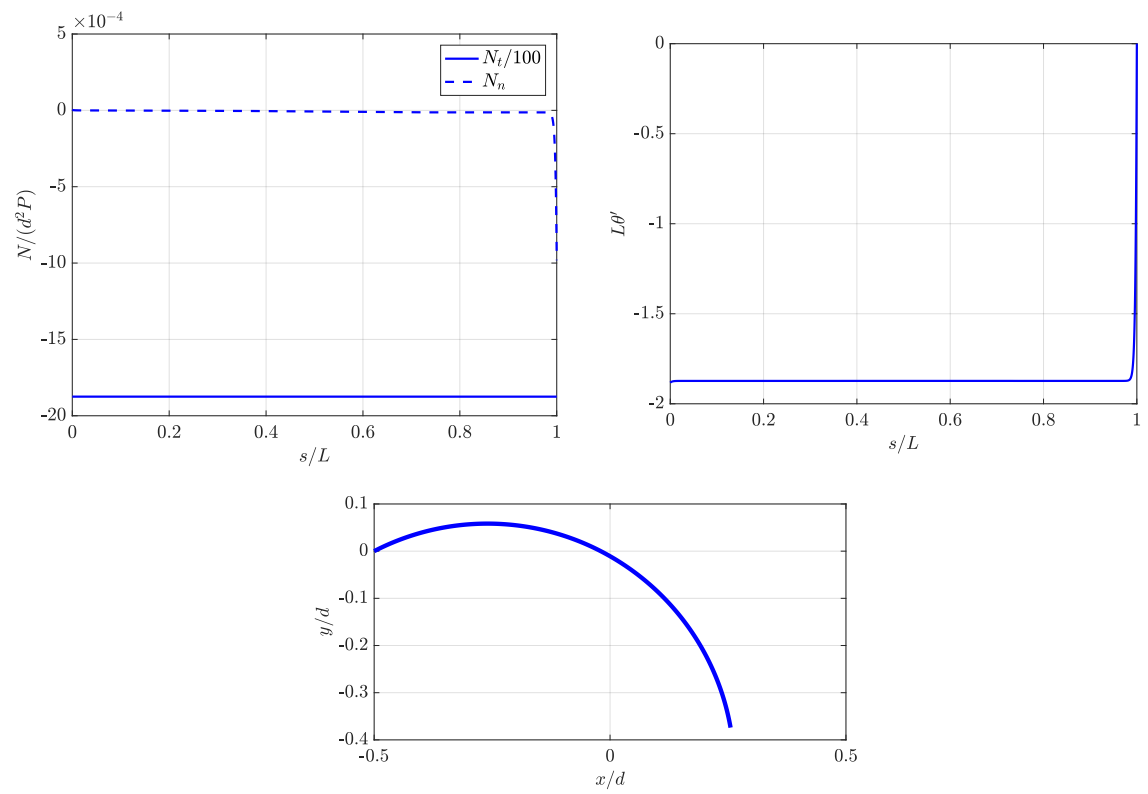

FIG. 3.3. Results of the numerical approximation of the equations of a single leaflet with $\beta=0.2$ and specific boundary condition $\theta(0)=-27.32^{\circ}$. The plots report the normal $\left(N_{n}\right)$ and tangential $\left(N_{t}\right)$ tensions, the dimensionless curvature $L \theta^{\prime}$, and the placement of the leaflet. The choice of a specific boundary angle suppresses the tensional peak at the left boundary.

The physiological values are listed on the second column of the table, while values obtained by the numerical integration of our model are written in the third and fourth columns (healthy state of Figure 3.5(left)). There is a satisfactory quantitative correspondence between the numerical and measured values except for the vertical coordinate of the triple point $h$. Since $P d^{2}>>\bar{k}$, the shape profile far from the boundaries is not significantly affected by the bending stiffness of the leaflets, but it is essentially dominated by the pressure. Indeed the circular shape of the leaflets (see Figure 3.5 ) is mainly determined by the balance between the pressure and the tension of the chordae tendineae, while the bending modulus has a negligible role far from the boundaries. This results from the dimensional analysis of section 3.1, where the parameter $\alpha$ accounts for the ratio between pressure and bending: $\alpha=3 \cdot 10^{5}$ means that the pressure dominates over bending.

Figure 3.6 plots the components of the reaction forces on the leaflets $\mathbf{N}_{1}$ and $\mathbf{N}_{2}$ versus the dimensionless curvilinear coordinate $\sigma=s / \bar{s}_{i}, i=1,2$. The normal component is much smaller than the tangential one except at the very endpoints of the rods, as discussed in section 1.2 for a single leaflet. The small normal component of the reactions $\mathbf{N}_{i}$ takes a geometrical meaning when considering (1.16a): the quantity $k(s) \theta^{\prime}(s)$ is almost constant, thus yielding that the curvature $\theta^{\prime}$ nearly behaves like the inverse of the bending modulus $k(s)$.

The tensional boundary layers at the annulus can be suppressed also in the case of a full mitral valve by a suitable prescription of the angle at the endpoint. In Figure 3.7 are the reported results of the numerical integration of the mitral valve for $\alpha=3 \cdot 10^{5}$ and $\beta=0.2$, with boundary conditions at the annulus $\theta_{1}(0)=11.12^{\circ}$ and $\theta_{2}(0)=0.5^{\circ}$. 

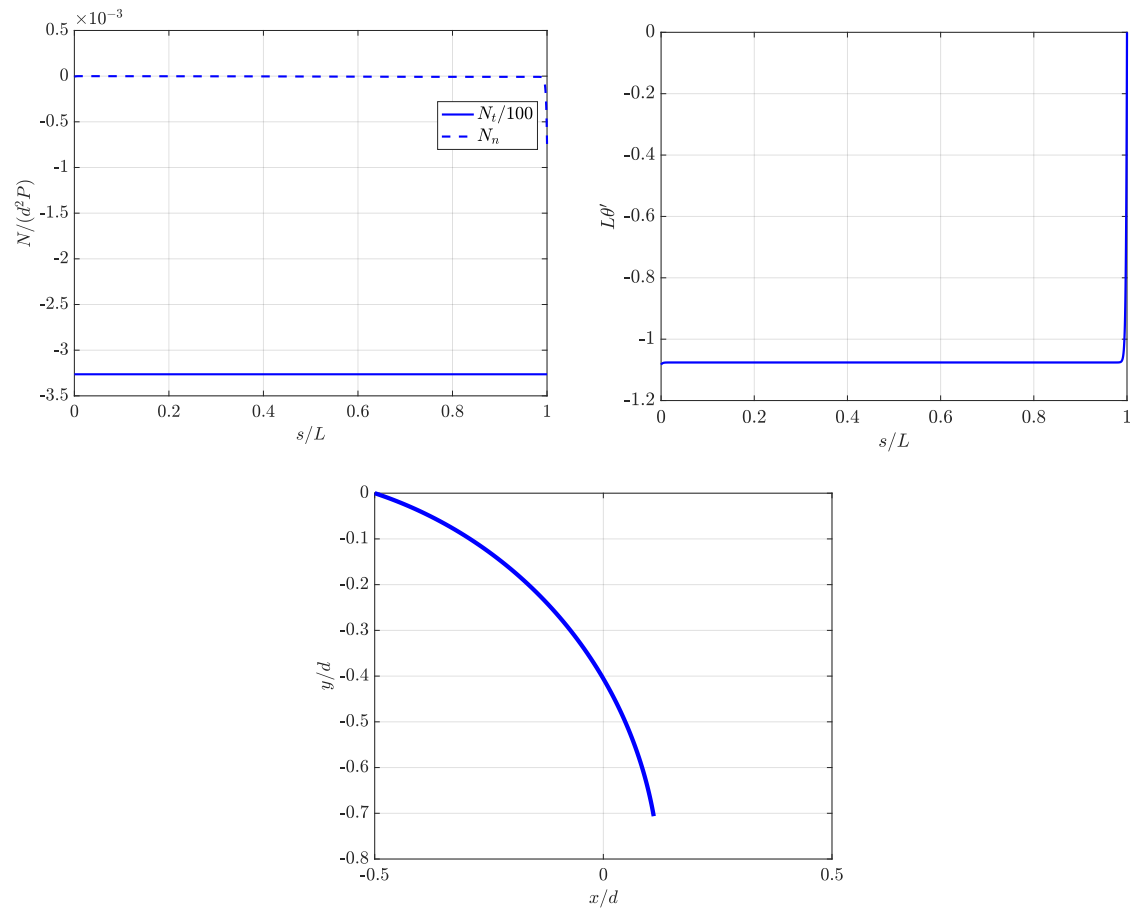

FIG. 3.4. Numerical integration of the equations for a single leaflet performed with $\beta=0.32$ imposing the specific boundary conditions $\theta(0)=18.35^{\circ}$. The plots report the normal $\left(N_{n}\right)$ and tangential $\left(N_{t}\right)$ tensions, the dimensionless curvature $L \theta^{\prime}$, and the profile of the leaflet in the plane. The choice of a specific boundary angle suppresses the tensional peak at the left boundary.
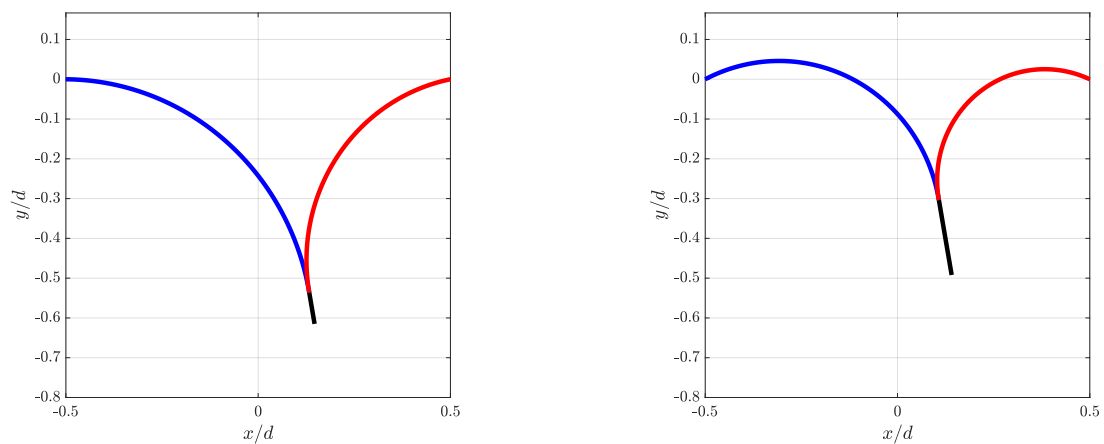

FIG. 3.5. Position of the leaflets of the mitral valve with kinematics constrained by the elongation of the chordae tendineae. Both numerical simulations correspond to $\alpha=3 \cdot 10^{5}, \gamma_{1}=0.8$, and $\gamma_{2}=0.98$, while $\beta=0.2$ (left) and $\beta=0.1$ (right). The chordae tendineae have the correct physiological length (left), or they are too long to prevent the prolapse of the mitral valve (right).

3.4. Sensitivity analysis. From an applicative point of view, it is important to consider the sensitivity of the results to variations of parameters that cannot be accurately determined. In our case, while pressure and stiffness ( $\alpha$ and $\beta$, respectively) are well known in an healthy patient from medical and soft biomechanics studies, the knowledge of the curvilinear coordinates of the early contact point is to be determined 
TABLE 3.1

Physiological and numerical values of the angles that the leaflets make with the annular plane in the hinge points.

\begin{tabular}{|c|c|c|c|c|}
\hline & \multicolumn{2}{|c|}{ Physiological values } & \multicolumn{2}{c|}{ Numerical values } \\
\hline Leaflet angles & Right: $\phi_{1}$ & Left: $\phi_{2}$ & Right: $\phi_{1}$ & Left: $\phi_{2}$ \\
\hline & $37^{\circ}$ & $21^{\circ}$ & $31^{\circ}$ & $18^{\circ}$ \\
\hline $\bar{s}_{1} / d$ & 0.5 & \multicolumn{2}{c|}{0.72} \\
\hline $\bar{s}_{2} / d$ & 0.67 & \multicolumn{2}{c|}{0.53} \\
\hline$h / d$ & 0.26 & \multicolumn{2}{c}{} \\
\hline
\end{tabular}
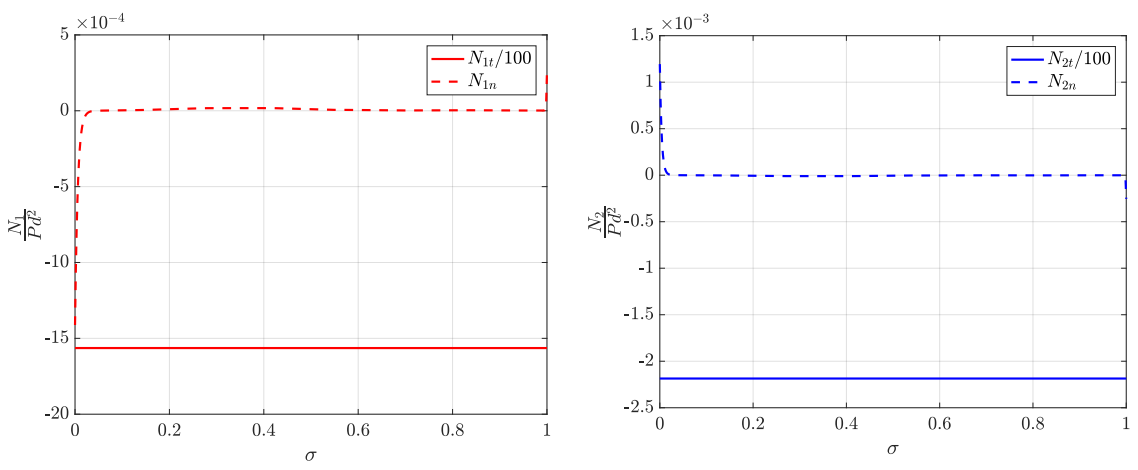

FIG. 3.6. Tangential and normal components of the tensions $\mathbf{N}_{1}$ (left) and $\mathbf{N}_{2}$ (right) obtained with $\alpha=3 \cdot 10^{5}, \beta=0.2$, and boundary condition $\theta^{\prime}(0)=0$. The normal component is negligible versus the tangential one.
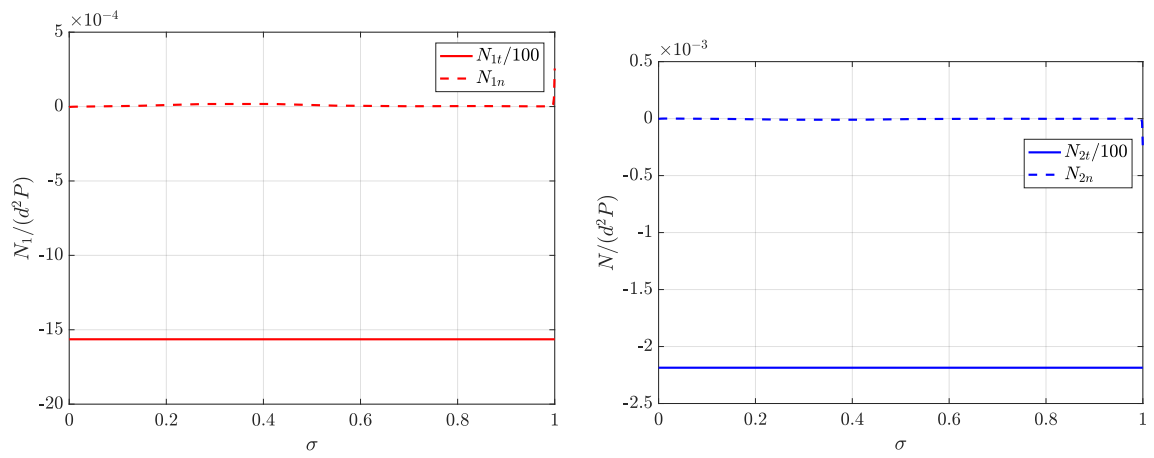

FiG. 3.7. Tangential and normal tension for the leaflets 1 (left) and 2 (right), obtained with $\alpha=3 \cdot 10^{5}, \beta=0.2$, and boundary conditions on the initial angle of each leaflet $\theta_{1}(0)=11.12^{\circ}$ and $\theta_{2}(0)=0.5^{\circ}$. The choice of these specific boundary conditions prevent the emergence of stress boundary layers at the annulus.

(see Remark 1). We are therefore interested in exploring numerically how the simulation results, reported in Table 3.1, are affected by variations of the parameters $\gamma_{1}$ and $\gamma_{2}$, while keeping all the other parameters fixed. To this aim, we perform a series of simulations with different values of the parameters $\gamma_{1}$ and $\gamma_{2}$

The results are reported in Tables 3.2 and 3.3. It is apparent that, while the increase of $\gamma_{1}$ and $\gamma_{2}$ up to $10 \%$ affects $\bar{s}_{1} / d$ and $\bar{s}_{2} / d$ and the angles, it has almost no effects on $h / d$. This means that the $y$ coordinate of the contact point is largely independent from the parameters $\gamma_{1}$ and $\gamma_{2}$.

Copyright $@$ by SIAM. Unauthorized reproduction of this article is prohibited. 
TABLE 3.2

Numerical values of the tangent angle at the fixed point ( $\phi_{1}$ and $\left.\phi_{2}\right)$, curvilinear cooordinate of the contact point $\left(\bar{s}_{1} / d\right.$ and $\left.\bar{s}_{2} / d\right)$, and depth of the contact point $h / d$ for different values of $\gamma_{1}$.

\begin{tabular}{|c|c|c|c|c|c|}
\hline & \multicolumn{2}{|c|}{ Leaflet angles } & \multicolumn{2}{c|}{ Contact coordinate } & Depth \\
\hline$\gamma_{1}$ & $\phi_{1}$ & $\phi_{2}$ & $\bar{s}_{1} / d$ & $\bar{s}_{2} / d$ & $h / d$ \\
\hline 0.7 & $37^{\circ}$ & $14^{\circ}$ & 0.67 & 0.95 & 0.53 \\
\hline 0.75 & $34^{\circ}$ & $16^{\circ}$ & 0.69 & 0.92 & 0.53 \\
\hline 0.8 & $31^{\circ}$ & $18^{\circ}$ & 0.72 & 0.9 & 0.53 \\
\hline 0.85 & $29^{\circ}$ & $20^{\circ}$ & 0.74 & 0.87 & 0.53 \\
\hline
\end{tabular}

TABLE 3.3

Numerical values of the tangent angle at the fixed point ( $\phi_{1}$ and $\left.\phi_{2}\right)$, curvilinear cooordinate of the contact point $\left(\bar{s}_{1} / d\right.$ and $\left.\bar{s}_{2} / d\right)$ and depth of the contact point $h / d$ for different values of $\gamma_{2}$.

\begin{tabular}{|c|c|c|c|c|c|}
\hline & \multicolumn{2}{|c|}{ Leaflet angles } & \multicolumn{2}{c|}{ Contact coordinate } & Depth \\
\hline$\gamma_{2}$ & $\phi_{1}$ & $\phi_{2}$ & $\bar{s}_{1} / d$ & $\bar{s}_{2} / d$ & $h / d$ \\
\hline 0.9 & $27^{\circ}$ & $22^{\circ}$ & 0.76 & 0.86 & 0.53 \\
\hline 0.95 & $30^{\circ}$ & $20^{\circ}$ & 0.73 & 0.88 & 0.53 \\
\hline 0.98 & $31^{\circ}$ & $18^{\circ}$ & 0.72 & 0.9 & 0.53 \\
\hline 1 & $32^{\circ}$ & $17^{\circ}$ & 0.71 & 0.91 & 0.53 \\
\hline
\end{tabular}

TABLE 3.4

Numerical values of the tangent angle at the fixed point $\left(\phi_{1}\right.$ and $\left.\phi_{2}\right)$, curvilinear cooordinate of the contact point $\left(\bar{s}_{1} / d, \bar{s}_{2} / d\right.$, and $\left.\left(s_{3}\right) / d\right)$, and depth of the contact point $h / d$ for different values of $\gamma_{1}$ and $\gamma_{2}$.

\begin{tabular}{|c|c|c|c|c|c|c|c|}
\hline & \multicolumn{2}{|c|}{ Leaflets angles } & \multicolumn{3}{|c|}{ Contact coordinate } & Depth \\
\hline$\gamma_{1}$ & $\gamma_{2}$ & $\phi_{1}$ & $\phi_{2}$ & $\bar{s}_{1} / d$ & $\bar{s}_{2} / d$ & $\bar{s}_{3} / d$ & $h / d$ \\
\hline 0.75 & 0.91 & $30^{\circ}$ & $19^{\circ}$ & 0.73 & 0.89 & 0.02 & 0.53 \\
\hline 0.8 & 0.98 & $31^{\circ}$ & $18^{\circ}$ & 0.72 & 0.9 & 0.08 & 0.53 \\
\hline 0.92 & 1.2 & $37^{\circ}$ & $14^{\circ}$ & 0.67 & 0.95 & 0.24 & 0.53 \\
\hline
\end{tabular}

By contrast, in Table 3.4 are the reported results obtained by increasing both $\gamma_{1}$ and $\gamma_{2}$, while keeping the ratio $\gamma_{1} / \gamma_{2}$ constant. The angle of the right rod increases, while the angle of the left rod decreases. Accordingly $\bar{s}_{1} / d$ decreases and $\bar{s}_{2} / d$ increases as well as $\bar{s}_{3} / d$, while $h / d$ still remains constant. The fact that $h / d$ remains constant means that it is mainly determined by the parameter $\beta$ which fixes the curvature of the leaflets.

Although far from the boundaries the solution is simply defined by two crossing circles; one should not infer that the full solution of the mechanical system is trivially the intersection of two circles because it is completely defined by other two unknowns: the curvilinear coordinate of the attachment point and the detachment angle of each leafleat from the annulus that stem from the complex nonlinear interactions of the system.

3.5. A mechanical remark and an embryological conjecture. The perturbative analysis of section 2 shows that, in the high pressure regime of interest, along the single flap almost everywhere the tension is essentially equal to the force $q$ exerted by the chordae tendineae at the free endpoint. The numerical results for the complete valve show that this is not true when the rods are in contact: the plots of tension of Figures 3.1 and 3.6 are obtained for the same values of the parameters $\alpha$ and $\beta$, but the computed values of $N_{t}$ are completely different. As a matter of fact, the contact between the rods makes the tensional dynamics more complex: while the tensional balance (1.19) holds in the triple point, its split in the single rods cannot be easily determined without addressing the whole differential problem. 
The sharp difference in tension that we predict in a single flap versus a full valve with leaflets in contact might have an embryological meaning. The study of the single leaflet of section 3.2 and of the full mature valve of section 3.3 are not only of theoretical interest because one system is the other one without contact but also because the latter system is embryologically the primordial state of the former. On the basis of such a remark, we have the possibility to argue about a possible mechanical role of tension in the morphogenesis of the mitral valve [15].

A longstanding morphological question is how the organs know that they have reached the right size [17]. In some cases the diffusion decay of morphogens, a process characterized by a decay length, plays a role. In some other cases the mechanical tension can provide an immediate and global transmission of information about a crucial event that is intrinsically related with the achievement of the final functional length. On the basis of our theoretical results, it is quite natural to hypothesize that a regulation of cell duplication can be based on the trigger of tensional value that can be produced only by the mutual contact of flaps. A comparison between Figure 3.6(left) and Figure 3.1(right) reveals that the tangent tension differs in the mature and immature system. This difference emerges after the contact between flaps. Experiments will be of course necessary in the future to validate such a conjecture.

4. Final remarks. A large body of literature exists on the numerical models for the mitral valve, in its full three-dimensional time-dependent interaction with the flow field, when the valve is represented as a structure immersed in the blood flow (see [18] as an example). In this work we have followed a different approach that is almost unexplored in the literature (a remarkable exception is [5]). We have considered a bare-bones model where the dimensionality of the problem is reduced to the planar case, the large aspect ratio of the leaflet is enforced representing it as a rod, and we have focused on the static balance between blood pressure, tension in the leaflets, and tension of the chordae tendineae. The bidimensional model of the atrial valve obtained insofar has been rigorously deduced and illustrated both in its mature configuration, at systolic closure, and its primordial form, when atrioventricular cushions remodel to form the valve leaflets. The simplicity of the mathematical model, while derived on the basis of rigorous arguments, provides an extremely flexible tool that can be fully controlled in terms of the bending modulus, the early contact point between leaflets, the coordinates of the papillary muscles, and the length of the chordae tendineae. Outputs of the numerical simulations are quantities of routine clinical interest like the deflection angle of the leaflets from the annular plane and the location of the first contact point between the flaps. One can notice that even though in our model we suppose the tension of the chordae tendineae to be known, we can recast the problem supposing to know the physiological length of the wires and from that compute their tension.

The flexibility of the model has two main consequences. The equations can be numerically integrated in less than one second on a laptop, thus providing a potentially immediate clinical support to surgeons in their practice to design the shape of the leaflets or the length of the chords on the basis of the elementary patient's morphology. The computational cost is so low that a very large number of surgery solutions could be explored in real time.

The simplicity of the model also offers the possibility to address more basic science questions as related to the valvular morphogenesis. This issue has been investigated in the past literature in terms of purely fluid dynamical solicitations [3]. The most 
ambitious study of the valvulogenesis process has been addressed by Buskohl, Jenkins, and Butcher [6]: they adopt a two-dimensional (three-dimensional axial-symmetric) representation of the cushion, and they numerically solve both the blood flow and the coupled elastic structural problem of the cushion. Endowing the material evolution of a simple growth law, they numerically demonstrate that a physiological evolution of the cushion can be predicted on the basis of a purely mechanical stimulus. Our work is somehow complementary to theirs: our rod model applies only in the late stage of the morphogenetic process when the cushion has largely remodeled in terms of leaflets and chordae tendineae. In such a regime, we can compare the stress in the flap before and after closure. The computed large difference in stress, due to the large difference in pressure between the open- and closed-valve regime, might support an argument in favor of a mechanobiological control of the growth of the mitral valve. Our model could potentially be an agile theoretical tool to support the study of the origin of congenital cardiac defects [10].

\section{REFERENCES}

[1] T. Arts, S. Meerbaum, R. Reneman, and E. Corday, Stresses in the closed mitral valve: A model study, J. Biomech., 16 (1983), pp. 539-547.

[2] C. M. Bender And S. A. Orszag, Advanced Mathematical Methods for Scientists and Engineers I: Asymptotic Methods and Perturbation Theory, Springer, New York, 2013.

[3] S. V. Biechler, J. D. Potts, M. J. Yost, L. Junor, R. L. Goodwin, and J. W. Weidner, Mathematical modeling of flow-generated forces in an in vitro system of cardiac valve development, Ann. Biomed. Engrg., 38 (2010), pp. 109-117.

[4] B. Brazile, B. Wang, G. Wang, R. Bertucci, R. Prabhu, S. S. Patnaik, J. R. Butler, A. Claude, E. Brinkman-Ferguson, L. N. Williams and J. Liao, On the bending properties of porcine mitral, tricuspid, aortic, and pulmonary valve leaflets, J. Long-Term Eff. Med. Implants, 25 (2015), pp. 41-53.

[5] M. BRyhn AND L. GaRding, The mitral valve mechanism with normal and prolapsed leaflets in the light of a dynamic model, Clin. Cardiol., 9 (1986), pp. 483-486.

[6] P. R. Buskohl, J. T. Jenkins, and J. T. Butcher, Computational simulation of hemodynamic-driven growth and remodeling of embryonic atrioventricular valves, Biomech. Model. Mechanobiol., 11 (2012), pp. 1205-1217.

[7] L. Deorsola And A. Bellone, The golden proportion in the scallop geometry of normal mitral valves. when nature plays with jigsaw puzzles, Echocardiography, 36 (2019), pp. 1028-1034.

[8] H. Gao, N. QI, L. Feng, X. Ma, M. Danton, C. Berry, and X. Luo, Modelling mitral valvular dynamics-current trend and future directions, Int. J. Numer. Methods Biomed. Eng., 33 (2017), p. e2858.

[9] S. Goenezen, M. Y. Rennie, and S. Rugonyi, Biomechanics of early cardiac development, Biomech. Model. Mechanobiol., 11 (2012), pp. 1187-1204.

[10] W. J. Kowalski, K. Pekkan, J. P. Tinney, and B. B. Keller, Investigating developmental cardiovascular biomechanics and the origins of congenital heart defects, Front. Physiol., 5 (2014), p. 408.

[11] M. Misfeld And H.-H. Sievers, Heart valve macro-and microstructure, Philos. Trans. Roy. Soc. London Ser. B, 362 (2007), pp. 1421-1436.

[12] G. Napoli and A. Goriely, A tale of two nested elastic rings, Proc. A, 473 (2017), 20170340.

[13] D. Oliveira, J. Srinivasan, D. Espino, K. Buchan, D. Dawson, and D. Shepherd, Geometric description for the anatomy of the mitral valve: A review, J. Anat., 237 (2020), pp. 209-224.

[14] S. Rodbard, Vascular modifications induced by flow, Am. Heart J., 51 (1956), pp. 926-942.

[15] E. Steed, F. Boselli, And J. Vermot, Hemodynamics driven cardiac valve morphogenesis, Biochim. Biophys. Acta Mol. Cell Res., 1863 (2016), pp. 1760-1766.

[16] S. Turzi, M. Zoppello, AND D. AmBrosi, Equilibrium of two rods in contact under pressure, Quart. J. Mech. Appl. Math., 73 (2020), pp. 329-346.

[17] G. Vogel, How do organs know when they have reached the right size?, Science, 340 (2013), pp. 1156-1157, https://doi.org/10.1126/science.340.6137.1156-b. 
[18] E. Votta, E. Caiani, F. Veronesi, M. Soncini, F. M. Montevecchi, and A. Redaelli, Mitral valve finite-element modelling from ultrasound data: A pilot study for a new approach to understand mitral function and clinical scenarios, Philos. Trans. Roy. Soc. A, 366 (2008), pp. 3411-3434.

[19] E. J. Weinberg, D. Shahmirzadi, And M. R. K. Mofrad, On the multiscale modeling of heart valve biomechanics in health and disease, Biomech. Model. Mechanobiol., 9 (2010), pp. 373-387.

Copyright (c) by SIAM. Unauthorized reproduction of this article is prohibited. 